\title{
Long-term efficacy and safety of SARS- CoV-2 vaccination in patients with chronic kidney disease, on dialysis or after kidney transplantation: a national prospective observational cohort study
}

P. Bouwmans ${ }^{1,2^{*}}$, , A. L. Messchendorp ${ }^{3}$, J. S. Sanders ${ }^{3}$, L. Hilbrands ${ }^{4}$, M. E. J. Reinders ${ }^{5}$, P. Vart ${ }^{6,7}$, F. J. Bemelman ${ }^{8}$, A. C. Abrahams ${ }^{9}$, M. A. van den Dorpel ${ }^{10}$, M. A. Ten Dam ${ }^{11}$, A. P. J. de Vries ${ }^{12}$, T. Rispens ${ }^{13,14}$, M. Steenhuis ${ }^{13,14}$, R. T. Gansevoort ${ }^{3}$, M. H. Hemmelder ${ }^{1,2}$ and RECOVAC Collaborators

\begin{abstract}
Background: COVID-19 is associated with increased morbidity and mortality in patients with chronic kidney disease (CKD) stages G4-G5, on dialysis or after kidney transplantation (kidney replacement therapy, KRT). SARS-CoV-2 vaccine trials do not elucidate if SARS-CoV-2 vaccination is effective in these patients. Vaccination against other viruses is known to be less effective in kidney patients. Our objective is to assess the efficacy and safety of various types of SARS-CoV-2 vaccinations in patients with CKD stages G4-G5 or on KRT.

Methods: In this national prospective observational cohort study we will follow patients with CKD stages G4-G5 or on KRT $(n=12,000)$ after SARS-CoV-2 vaccination according to the Dutch vaccination program. Blood will be drawn for antibody response measurements at day 28 and month 6 after completion of vaccination. Patient characteristics and outcomes will be extracted from registration data and questionnaires during 2 years of follow-up. Results will be compared with a control group of non-vaccinated patients. The level of antibody response to vaccination will be assessed in subgroups to predict protection against COVID-19 breakthrough infection.

Results: The primary endpoint is efficacy of SARS-CoV-2 vaccination determined as the incidence of COVID-19 after vaccination. Secondary endpoints are the antibody based immune response at 28 days after vaccination, the durability of this response at 6 months after vaccination, mortality and (serious) adverse events.
\end{abstract}

Conclusion: This study will fulfil the lack of knowledge on efficacy and safety of SARS-CoV-2 vaccination in patients with CKD stages G4-G5 or on KRT.

Trial registration: The study protocol has been registered in clinicaltrials.gov(NCT04841785).

\section{Current knowledge about this subject}

*Correspondence: p.bouwmans@maastrichtuniversity.nl

${ }^{2}$ CARIM school for cardiovascular disease, University of Maastricht,

Maastricht, the Netherlands

Full list of author information is available at the end of the article

(c) The Author(s) 2022. Open Access This article is licensed under a Creative Commons Attribution 4.0 International License, which permits use, sharing, adaptation, distribution and reproduction in any medium or format, as long as you give appropriate credit to the original author(s) and the source, provide a link to the Creative Commons licence, and indicate if changes were made. The images or other third party material in this article are included in the article's Creative Commons licence, unless indicated otherwise in a credit line to the material. If material is not included in the article's Creative Commons licence and your intended use is not permitted by statutory regulation or exceeds the permitted use, you will need to obtain permission directly from the copyright holder. To view a copy of this licence, visit http://creativecommons.org/licenses/by/4.0/. The Creative Commons Public Domain Dedication waiver (http://creativeco mmons.org/publicdomain/zero/1.0/) applies to the data made available in this article, unless otherwise stated in a credit line to the data. 
- COVID-19 has devastating impact on patients with CKD stages G4-G5, on dialysis or after kidney transplantation.

- Effective SARS-CoV-2 vaccination is very important in these vulnerable patient groups.

- Recent studies on vaccination in these patient groups are small short-term studies with surrogate endpoints.

\section{Contribution of this study}

- Assessment of incidence and course of COVID-19 after various types of SARS-CoV-2 vaccination during a twoyear follow-up period in not only patients on dialysis or kidney transplant recipients, but also in patients with CKD stages G4-G5.

- Quantitative analysis of antibody response after SARS-CoV-2 vaccination and its relationship with incidence and course of COVID-19 in patients with CKD stages G4-G5, on dialysis or after kidney transplantation compared with a control group.

- Monitoring of (serious) adverse events and development of anti-HLA antibodies.

\section{Impact on practice or policy}

- Publication of the study design contributes to harmonization of SARS-CoV-2 vaccine study

methodology in kidney patients at high-risk for severe COVID-19.

- Data on efficacy of SARS-CoV-2 vaccination in patients with CKD will provide guidance for future vaccination policy.

Keywords: SARS-CoV-2, Vaccine, Chronic kidney disease, Kidney transplantation, Dialysis, Safety, Efficacy, Antibody response

\section{Background}

The coronavirus disease 2019 (COVID-19) pandemic has great impact on all aspects of society and healthcare. Patients with a severely impaired kidney function (CKD stages G4-G5) and patients on dialysis or after kidney transplantation, referred to as kidney replacement therapy (KRT), were shown to be extremely vulnerable. The COVID-19 associated mortality risk in these patient groups was reported to be 3- to 4-fold increased as compared to the general healthy population [1], which is considerably higher than the 1.5-to-2-fold increase that was described in patients with obesity, hypertension or diabetes [2]. Data from the European Renal Association - European Dialysis and Transplant Association (ERAEDTA) COVID-19 database (ERACODA) show a high case fatality rate in patients on KRT [3, 4]. Therefore, the availability of an effective and safe vaccine will be extremely important for patients with CKD stages G4-G5 or on KRT.

Recently, efficacy and safety of different SARS-CoV-2 vaccine types have been demonstrated to be high in the general population [5-8]. So far, patients with CKD stages G4-G5 or on KRT were not or in low numbers included in these trials [9]. It is well known that vaccination efficacy against other viruses such as hepatitis $B$ and influenza is considerably lower in patients with severely impaired kidney function, due to the immunosuppressive effect of uremic waste products [10]. A lesser antibody response after vaccination is to be expected in kidney transplant recipients due to the use of immunosuppressive agents. A recent Call to Action by the ERAEDTA Council and ERACODA Working Group included the advice to perform dedicated vaccination studies to address this important knowledge gap regarding the efficacy of SARS-CoV-2 vaccination [11]. Recent studies have demonstrated the humoral response following SARS-CoV-2 vaccination in patients on KRT. One study showed a robust humoral response in the majority of haemodialysis patients after SARS-CoV-2 vaccination [12], but in a different study when compared to a control group, antibody titres were significantly lower [13]. Another study demonstrated a significantly reduced antibody response in 34 solid organ transplantation patients compared to health care workers [14]. Current data on SARS-CoV-2 vaccination efficacy in kidney patients report on surrogate endpoints, such as antibody titres. 


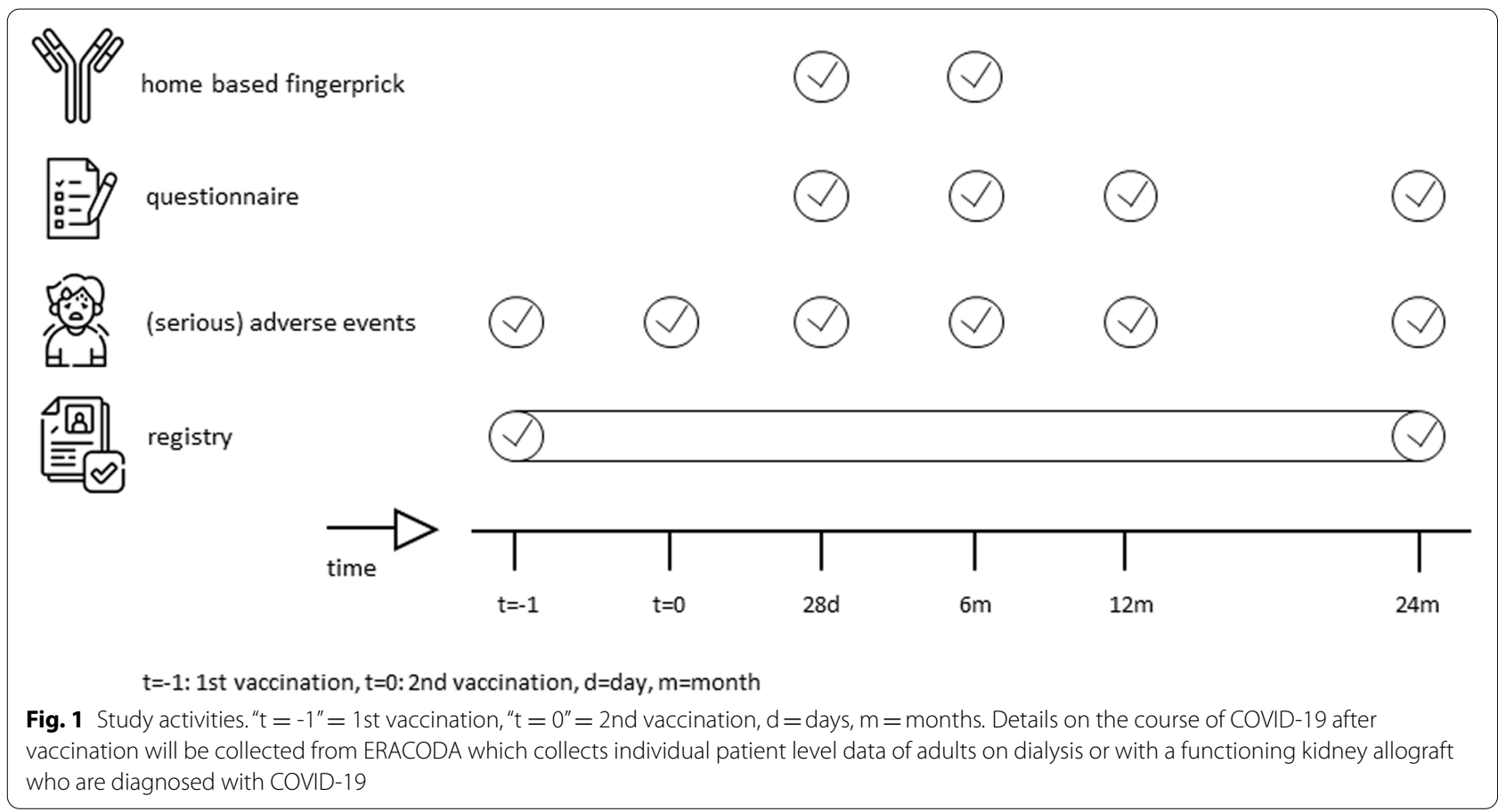

Clinical endpoints are currently lacking in the assessment of the efficacy of SARS-CoV-2 vaccination in kidney patients.

Therefore, we established the RECOVAC consortium (REnal patients COvid-19 VACcination) in the Netherlands with active participation of the Dutch Kidney Patients Association (NVN). This consortium will perform the Long-term Efficacy and Safety of SARS-CoV-2 vaccination (LESS CoV-2) study. In this study we aim to assess efficacy of SARS-CoV-2 vaccination in patients with CKD stages G4-G5 and on KRT in comparison to non-vaccinated control groups in a large-scale national registry study. We hypothesize that kidney patients obtain lesser protection from SARS-CoV-2 vaccination.

\section{Methods and analysis Study design}

This is a prospective national observational cohort study which includes all kidney patients who were vaccinated against SARS-CoV-2 in the Dutch vaccination programme. Currently available vaccines in the Netherlands are the mRNA-1273 (Moderna), BNT162b2mRNA (Pfizer), ChAdOx1 nCoV-19 (AstraZeneca) and Ad26.COV2.S (Janssen) vaccines [5-8]. Patients with CKD stages G4-G5 or on KRT received prioritization as medical high-risk group and predominantly received the mRNA-1273 vaccine in the Dutch vaccination programme. To assess the antibody dependent immune response after vaccination, blood samples will be collected at 28 days and at 6 months after the second vaccination with a home-based finger prick test. Antibody measurement will include both the receptor binding domain (RBD) of the spike (S) antigen and the nucleocapsid $(\mathrm{N})$ antigen [15]. Combining these antibody measurements allows to distinguish a natural infection from an antibody response after S1-based vaccination.

Questionnaires will be obtained during a two-year follow-up period to assess the incidence of COVID-19 or (serious) adverse events ((S)AEs) after vaccination. The study events are demonstrated in Fig. 1. The questionnaires are shown in the supplemental materials.

\section{Setting}

The study starts in April 2021 amidst the COVID-19 pandemic when the Dutch vaccination program has started to vaccinate high-risk patients, including patients with kidney disease. COVID-19 incidence rates are still high and the health care system has not yet regained full capacity to exert routine care to all patients, due to lack of capacity.

\section{Study participants}

The study population includes a subset of patients with CKD stages G4-G5 and patients on KRT in the Netherlands. The inclusion and exclusion criteria are mentioned in Table 1. Of note, patients will be eligible whether they have a history of COVID-19 or not. 
Table 1 Inclusion and exclusion criteria

Inclusion criteria

1. Age of $18-80$ years

2. Capable of understanding the purpose and risks of the study, to be fully informed and to give informed consent

3. Either

- eGFR $<30 \mathrm{ml} / \mathrm{min} / 1.73 \mathrm{~m}^{2}$ but not on dialysis or with a kidney transplant

- Haemodialysis or peritoneal dialysis

- Kidney transplant recipient at least 6 weeks after transplantation

Exclusion criteria $(\geq 1)$

-Patients who opted out for the national dialysis (RENINE) and kidney transplantation (NOTR) registries.

eGFR Estimated glomerular filtration rate

\section{Study enrolment}

All patients who previously gave informed consent in two national registries, for dialysis patients (RENINE) and kidney transplant recipients (NOTR), are selected for enrolment. Patients with CKD stages G4-G5 will be enrolled from a cohort of non-university hospitals (Santeon). All patients eligible to the registry will be followed to assess incidence of COVID-19 after vaccination, (S) $\mathrm{AEs}$ and vaccination status. Data of non-vaccinated patients which will be derived from RENINE and NOTR registries serve as control. Furthermore, data on vaccination efficacy in the general population, which will be derived from the National Institute for Public Health and the Environment (RIVM), serve as a control group.

For measurement of antibodies after completion of SARS-CoV-2 vaccination we intend to include a random subset of patients with CKD stages G4-G5 $(n=4000)$, patients on dialysis $(n=4000)$, and patients after kidney transplantation ( $n=4000)$ (Fig. 2). Patients older than 80 years are excluded from participation because they had priority in the Dutch national vaccination scheme over other high-risk groups. Consequently, they were vaccinated longer than 28 days ago, which is the accepted term in wherein finger-prick tests are obtained for antibody measurement.

\section{Informed consent for antibody measurement at home}

All patients eligible to antibody measurement at home are offered two options to give informed consent, namely a written and a digital informed consent. We invited patients to register for the study by e-mail, mail and by distribution of flyers in all participating hospitals. Patients could apply for a patient information folder being sent to their home address or they could access the information folder at the website by scanning a QR-code. The QR-code directed the patients to a digital platform where they could give a digital informed consent, signed by entering a code sent to their mobile phone. In addition, we used our study website [16] to provide online access to the digital platform where informed consent could be given. This procedure enables rapid information provision of patients with minimal effort for local health care workers in the hospitals.

\section{Patient characteristics}

The patient characteristics that will be obtained are listed in Table 2.

\section{Outcome definitions}

For an overview of the clinical endpoints in this study we refer to Table 3.

\section{Home based finger prick tests}

Blood samples will be obtained through self-obtained sample collection by use of a home-based finger prick test. The finger prick tests will be sent by mail to all participants. Written instructions are given to apply the finger prick test and how to return the finger prick tests. This procedure enables blood sampling without further need of effort from local health care workers in the hospitals. Only hemodialysis patients were offered to contact their dialysis center, when they encounter difficulties during the blood sample collection.

\section{Antibody measurement}

We will analyse the presence of antibodies against the RBD of the SARS-CoV-2 S-protein (IgG anti-RBD antibody) using the Sanquin anti-SARS-CoV-2 RBD IgG ELISA assay [15]. This is an indirect ELISA using microtiter plates coated with RBD and detection by monoclonal mouse anti-human IgG. The antibodies against the RBD are the primary constituent of the humoral immune response after SARS-CoV-2 vaccination. We combine this test with a Sanquin anti-SARS-CoV-2 nucleocapsid protein (NP) bridging ELISA. This test is an indirect ELISA using microtiter plates coated with NP and detection by biotin-labelled NP. Both antibodies against RBD and NP arise after a natural COVID19 infection. In contrast, vaccination only induces antibodies to RBD, since NP is not part of any of the 


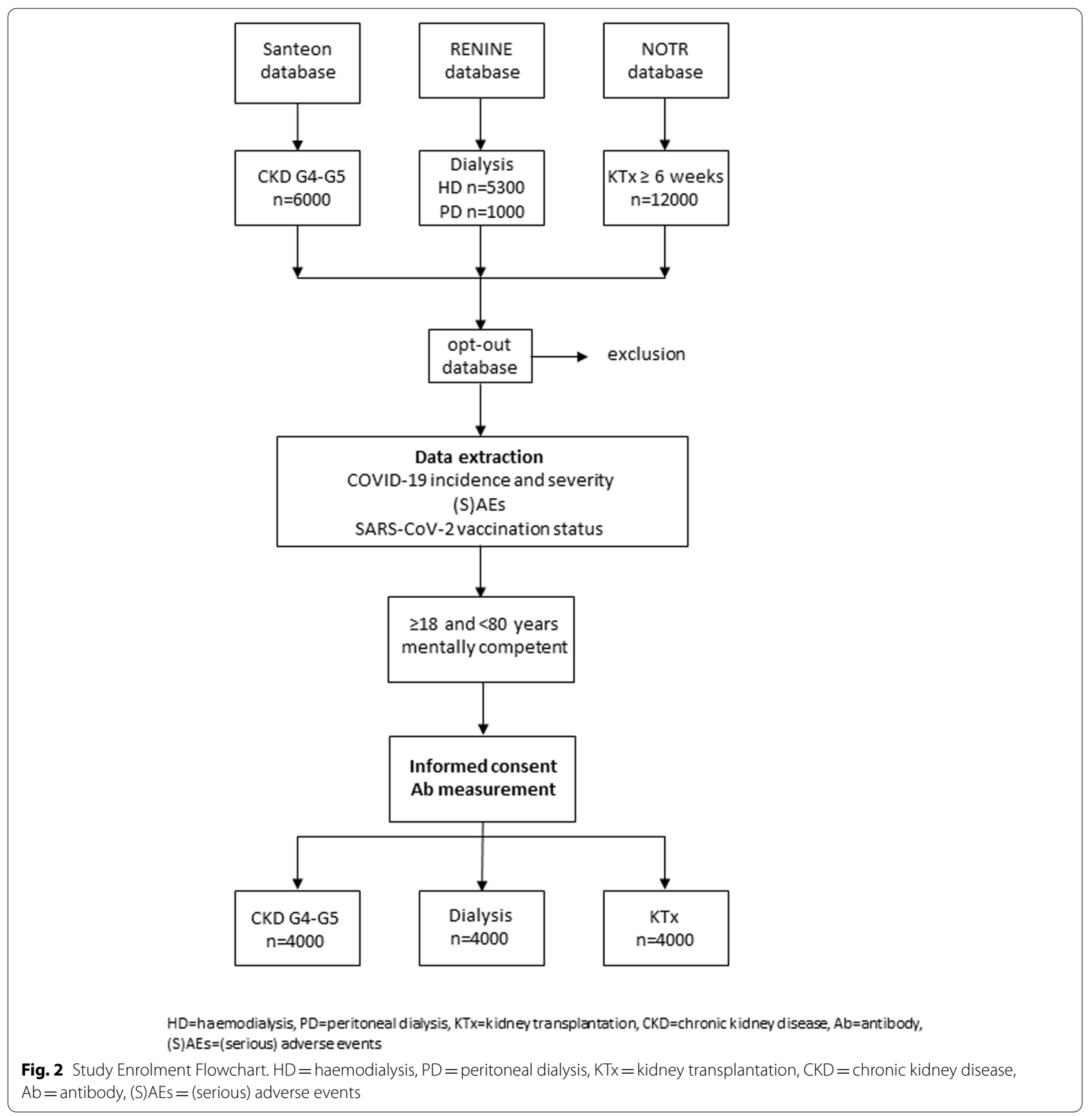

currently used vaccines. Combining these two tests enables us to differentiate between antibodies after vaccination or after previous COVID-19 infection.

\section{SARS-CoV-2 genotype variants}

We intend to collect data on SARS-CoV-2 genotype variance in our study population if available from clinical practice. We aim to assess the efficacy of the various
SARS-CoV-2 vaccine types used in our study population for protection against different SARS-CoV-2 strains.

\section{Statistical considerations}

Baseline characteristics of vaccinated patients with CKD stages G4-G5 or on KRT will be compared with non-vaccinated patients with CKD stages G4-G5 or on KRT and the vaccinated general population using a two-sample 
Table 2 Patient baseline characteristics

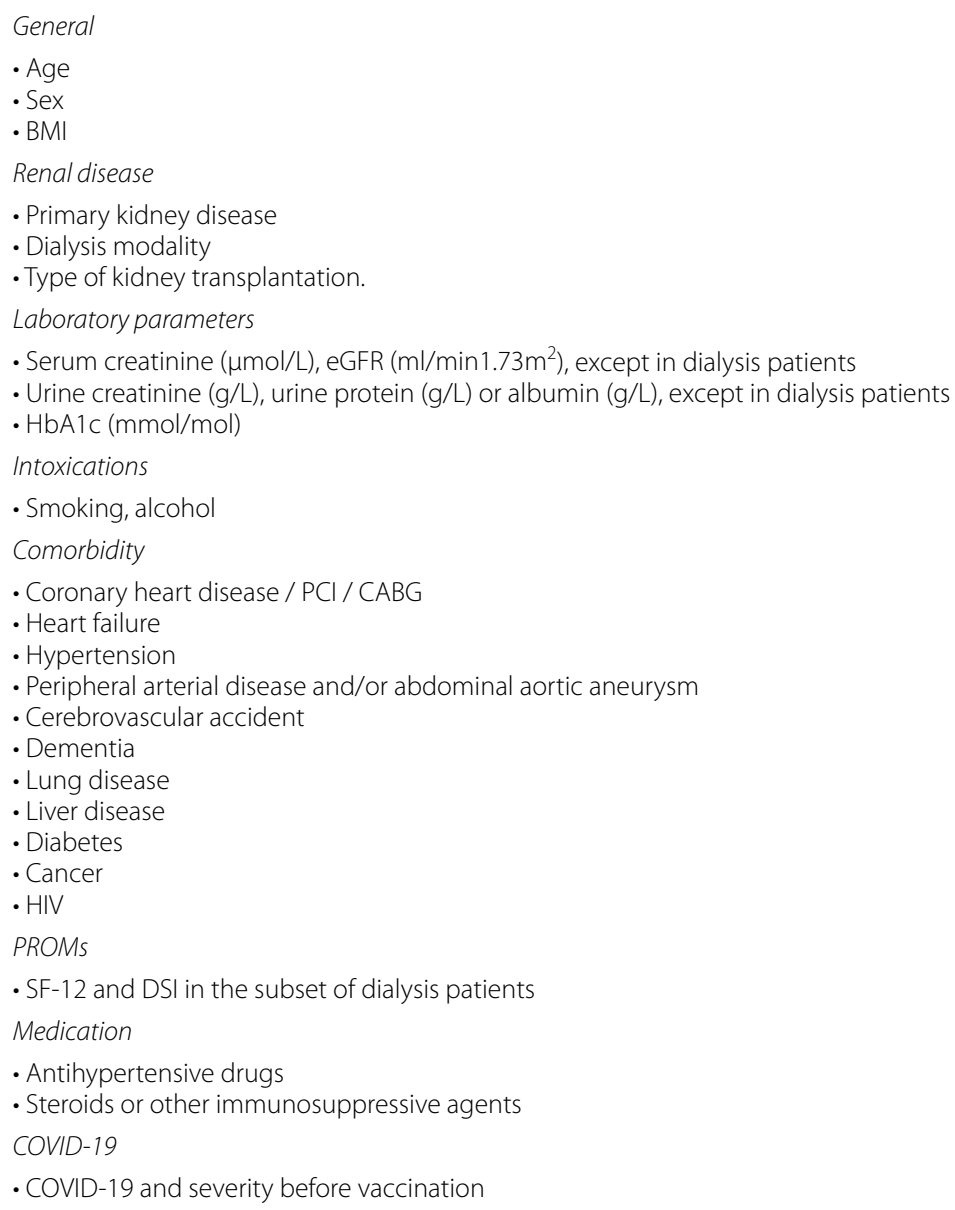

BMI Body Mass Index, eGFR estimated Glomerular Filtration Rate, HbA1c Haemoglobin A1c, PCI Percutaneous Coronary Intervention, CABG Coronary Arterial Bypass Grafting, HIV Human Immunodeficiency Virus, PROMs Patient Reported Outcome Measures, SF-12 12-item Short Form health survey, DSI Dialysis symptom index, COVID-19 Coronavirus disease 2019

t-test in case of continuous variables (or a Mann-Whitney U-test if data are not normally distributed), and a Pearson Chi-2 test in case of categorical variables.

\section{Analysis of primary endpoint}

Incidence rates of COVID-19 within 2 years after vaccination will be reported for each study population separately and incidence rates will be compared. Primary comparisons are comparisons of COVID-19 incidence rates in vaccinated patients versus non-vaccinated patients and versus the vaccinated general population. These comparisons will be made separately for patients with CKD stages G4-G5 or on KRT. For comparison of incidence rates, we will calculate crude hazard ratios. Vaccine efficacy will be reported as $100 \times(1$ - hazard ratio). We will calculate model adjusted hazard ratios using multivariable Cox-regression, in case of imbalance in study populations regarding relevant characteristics, such as age, sex, comorbidity, kidney replacement modality and use of immunosuppressive agents. Cause specific hazard ratios will be estimated to account for competing risk of mortality. Starting date of followup will be the date of second vaccination in vaccinated patients. Starting date of follow-up in non-vaccinated patients will be the same date (or $+/-7$ days from date of vaccination) as date of vaccination in the matched vaccinated patients to account for changes in incidence of COVID-19 over time. Non-vaccinated patients will be censored at the day of vaccination in case they are vaccinated before the end of follow-up. The aforementioned analyses will be repeated in patients with and patients without evidence of prior infection. Among vaccinated patients, incidence rates will also be compared across the various vaccines that will be used. For this purpose, vaccinated patients with CKD stages G4-G5 or on KRT and general population again will be analysed separately. 
Table 3 Clinical endpoints LESS-CoV-2 study

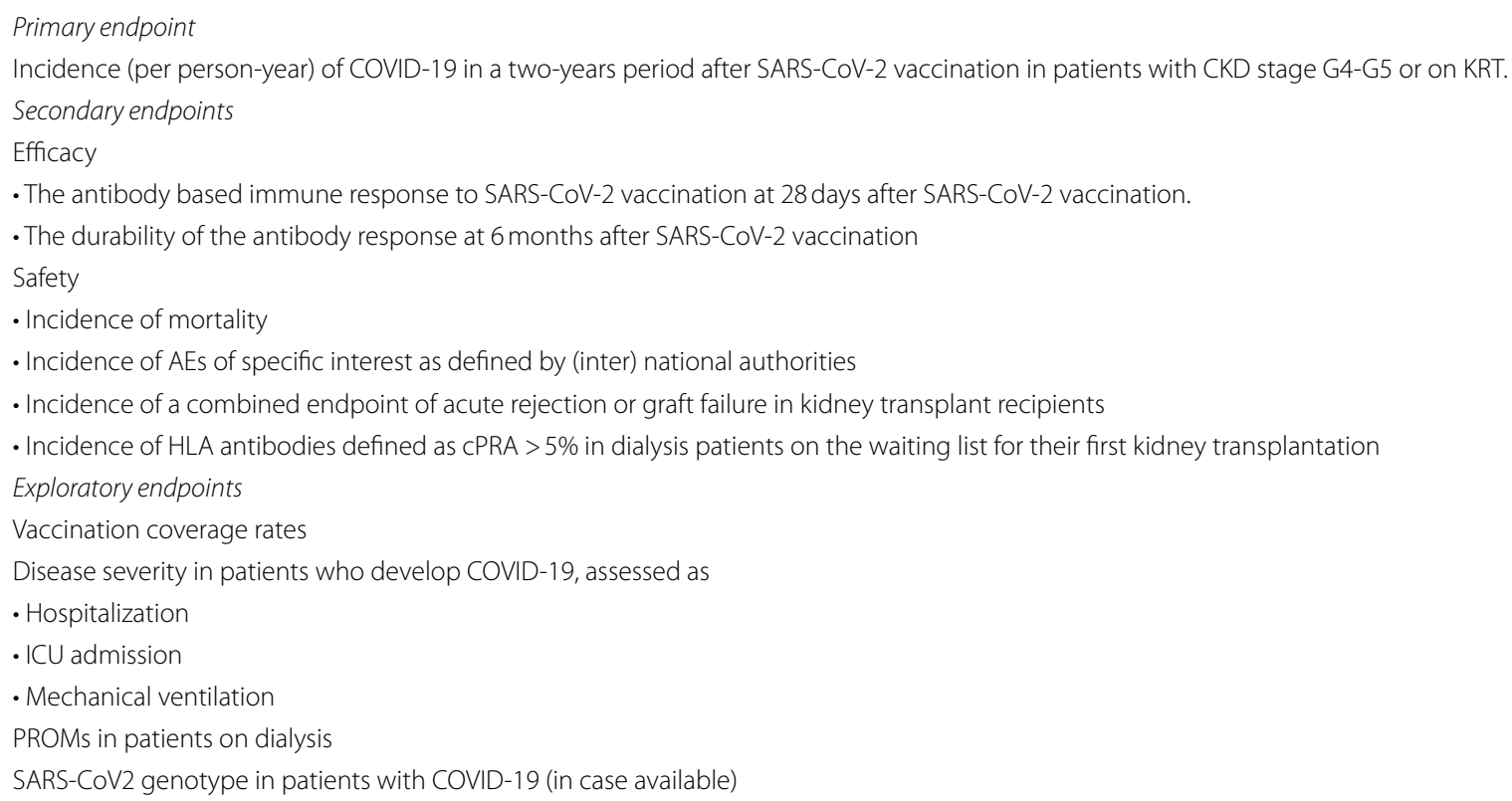

COVID-19 Coronavirus Disease 2019, SARS-CoV-2 Severe Acute Respiratory Syndrome Coronavirus-2, CKD Chronic Kidney Disease, KRT Kidney Replacement Therapy, AEs Adverse Events, HLA Human Leukocyte Antigen, CPRA Calculated Panel Reactive Antibody, ICU Intensive Care Unit, PROMs Patient Reported Outcome Measures

\section{Analysis of secondary endpoints}

Efficacy-Antibody response Participants will be classified as responders or non-responders [17]. Number and proportion of responders and non-responders at day 28 will be reported for each patient population. Pearson chi-2 test will be performed to assess whether antibody response differ between patient groups. In subsequent sensitivity analyses we will perform multiple regression analyses correcting for possible differences in age and sex between the patient groups. Additionally, in kidney transplant recipients we will investigate whether time after transplantation, and the different immunosuppressive regimens are associated with response rate.

To assess durability of response within each patient group, we will report and compare response at day 28 with response at month 6 after vaccination. Comparison will be made using McNemar test.

The association between antibody response after SARSCoV-2 vaccination and protection against COVID-19 will be examined for each patient group separately. For this, we will compare the antibody response at 28 days after vaccination in patients who develop COVID-19 with the antibody response in patients who did not develop COVID-19. Similar comparison will be made at 6 months after vaccination. We will examine this by use of the t-test or if more appropriate a non-parametric test. Subsequently, in a logistic regression model using Youden's index, a cut-off in the antibody response at 28 days will be defined to serve as a correlate of protection against COVID-19.

Safety Incidence rates of (S)AEs, mortality, acute rejection, graft failure or HLA antibodies will be calculated and compared across patient groups in analyses similar to analysis of the primary outcome.

\section{Discussion}

In the LESS-CoV-2 study we will assess the efficacy and safety of the mRNA-1273, BNT162b2, ChAdOx1 nCoV19 and Ad26.COV2.S SARS-CoV-2 vaccines in Dutch patients with CKD stages G4-G5, on dialysis or after kidney transplantation.

We expect to determine an antibody response cut-off value which allows to predict protection against COVID19 breakthrough infection after completion of SARS$\mathrm{CoV}-2$ vaccination. This contributes to the establishment of surrogate endpoints which will facilitate the development and approval of new vaccines [18]. In this study, we will identify non-responders by means of an insufficient antibody response after SARS-CoV-2 vaccination. This population can be offered a booster vaccine or an alternative vaccine to assess whether or not a sufficient antibody response can still be achieved. 
Furthermore, we will address the safety of SARS-CoV2-vaccination in patients with severe kidney insufficiency and in those that are treated with immunosuppressive drugs. Since these patients were not sufficiently represented in the large registration studies of the various vaccines, specific AEs associated with kidney failure and its treatment may not have been recognized so far. In addition, rare but possibly harmful AEs must be searched for in pharmacovigilance studies after widespread distribution of the vaccines. Recent reporting of vaccine-induced immune thrombotic thrombocytopenia caused by the ChAdOx1 nCoV-19 and Ad26.COV2.S SARS-CoV-2 vaccines is a possible example of such a rare complication [19]. It is essential to collaborate with large registry studies to be able to detect rare SAEs in patients with kidney disease. Different SARS-CoV-2 vaccines are administered to our subjects, and for that reason we will monitor the development of immunisation against HLA-antigens, allograft rejection and/or kidney transplant failure following SARS-CoV-2 vaccination.

Considering that items such as mental QoL, anxiety and depression may be positively influenced by vaccination, it is highly relevant to monitor the effect of SARS-CoV-2 vaccination on patient reported outcome measurements (PROMs). In our cohort of dialysis patients PROMs have been monitored since 2019. During the study we will further monitor the course of mental health of vaccinated and non-vaccinated dialysis patients and compare this to pre-COVID-19 pandemic era and the COVID-19 pandemic era without vaccines.

In our study we encountered the challenge to approach a large population to provide informed consent and obtain blood samples under high time pressure. We provided a variety of tools to overcome possible thresholds. First, the digital informed consent gave patients the opportunity to readily access study information and participation. An alternative option to apply for written informed consent was provided, thereby including patients that are not able to access digital platforms. Second by using a home-based finger prick test for antibody measurement, patients were not obliged to make travel expense to obtain blood samples and local health care workers were not burdened with more work during the pandemic. In addition, high-risk patient groups will not be exposed to an additional risk of contamination with SARS-CoV-2 due to extra visits to the hospitals. This strategy may attribute to a higher participation grade.

While our study has a real-life observational design, there are some relevant issues to mention which may impact the results. The reported efficacy depends on the presence of widespread contamination. If for any reason widespread contamination is halted, efficacy of vaccination might be confounded, hence less contamination will occur. The opposite could be the case in the midst of a pandemic where widespread contamination occurs. Additionally, participants are vaccinated according to the national Dutch vaccination programme, in which mRNA-1273 is predominantly administered to high-risk groups, including patients on dialysis or after kidney transplantation. Consequently, certain types of vaccines might be underrepresented. In this study we will only assess the humoral response following SARS-CoV-2 vaccination. Therefore, the immunogenicity of SARS-CoV-2 vaccination in high-risk kidney patients cannot be fully assessed.

Nonetheless, the LESS CoV-2 study will provide key information with regards to the efficacy of SARS$\mathrm{CoV}-2$ vaccines in high-risk kidney patients. Antibody response data and clinical endpoints will be collected in a large cohort of patients of whom patient characteristics uniformly are obtained in national registries with almost complete coverage. All high-risk kidney patient groups are represented in the study. As such, the study will be sufficiently powered to identify patients' characteristics and the humoral antibody response predisposing for COVID-19 breakthrough infection. Our study can serve as a template for other studies of vaccine efficacy in high-risk populations including the admission of digital informed consent and finger prick blood sampling at home. Harmonizing the design of vaccination studies will allow international comparison of larger datasets.

\section{Abbreviations}

Ab: Antibody; AEs: Adverse Events; BMI: Body Mass Index; CABG: Coronary Arterial Bypass Grafting; CKD: Chronic Kidney Disease; COVID-19: Coronavirus Disease 2019; cPRA: Calculated Panel Reactive Antibody; DSI: Dialysis Symptom Index; eGFR: Estimated Glomerular Filtration Rate; ELISA: Enzyme-Linked ImmunoSorbent Assay; ERACODA: European Renal Association COVID-19 Database; ERA-EDTA: European Renal Association - European Dialysis and Transplant Association; HbA1c: Haemoglobin A1c; HD: Haemodialysis; HIV: Human Immunodeficiency Virus; HLA: Human Leukocyte Antigens; ICU: Intensive Care Unit; IgG: Immunoglobulin G; KRT: Kidney Replacement Therapy; KTx: Kidney Transplantation; LAREB: Netherlands Pharmacovigilance Center; LESS CoV-2: Long term Efficacy and Safety of SARS-CoV-2 vaccination; mRNA: Messenger-RNA; N: Nucleocapsid; NFN: Dutch Federation of Nephrology; NOTR: Dutch kidney transplant recipient registration; NP: Nucleocapsid Protein; NTV: Dutch Transplant Society; NVN: Dutch Kidney Patient Association; PCl: Percutaneous Coronary Intervention; PD: Peritoneal Dialysis; PROMs: Patient-Reported Outcome Measures; QoL: Quality of Life; QR-code: Quick Response code; RBD: Receptor Binding Domain; RECOVAC: REnal patients COvid-19 VACcination; RENINE: Dutch dialysis patient registration; RIVM: Dutch National Institute for Public Health and the Environment; SAEs: Serious Adverse Events; SARS-CoV-2: Severe Acute Respiratory Syndrome Coronavirus-2; S: Spike; SD: Standard Deviation; SF-12: 12-item Short Form Health Survey 12; S1: Spike protein subunit 1; WMA: World Medical Association; WMO: World Medicine Organisation; ZonMw: The Netherlands Organisation for Health Research and Development.

\section{Supplementary Information}

The online version contains supplementary material available at https://doi. org/10.1186/s12882-022-02680-3. 
Additional file 1. Questionnaire at 1 month after vaccination.

Additional file 2. Questionnaires at 6,12 and 24 months after vaccination.

\section{Acknowledgements}

We would like to thank the RECOVAC collaborators Marcia L Kho, ${ }^{1}$ Debbie van Baarle, ${ }^{2}$ Renate G van der Molen, ${ }^{3}$ Carla C Baan, ${ }^{4}$ Dimitri A Diavatopoulos, ${ }^{5}$ Ester BM Remmerswall, ${ }^{6}$ Celine Imhof, ${ }^{7}$ Reshwan SRK Malahe, ${ }^{8}$ Sophie C Frölke, ${ }^{9}$ Nynke Rots, ${ }^{10}$ Fiona van der Klis, ${ }^{11}$ Ester ten Hoope, ${ }^{12}$ Wanda S Konijn, ${ }^{13}$ Tony de Ronde, ${ }^{14}$ Johanna PM Vervoort ${ }^{15}$ and Marion HJ Braks. ${ }^{16}$

${ }^{1}$ Erasmus Medical Center, Internal Medicine, Nephrology and Transplantation, Erasmus MC Transplant Institute. Rotterdam, the Netherlands.

${ }^{2}$ University Medical Center Groningen, Medical Microbiology and Infection Prevention. Groningen, the Netherlands.

${ }^{3}$ Radboud Institute for Molecular Life Sciences, Dept. of Laboratory Medicine, Laboratory of Medical Immunology, Radboud University Medical Center. Nijmegen, The Netherlands.

${ }^{4}$ Erasmus Medical Center, Internal Medicine, Nephrology and Transplantation, Erasmus MC Transplant Institute. Rotterdam, the Netherlands.

${ }^{5}$ Radboud Institute for Molecular Life Sciences; Dept. of Laboratory Medicine, Laboratory of Medical Immunology, section Pediatric Infectious Diseases; Radboud Center for Infectious Diseases, Radboud University Nijmegen Medical Center, Nijmegen, The Netherlands.

${ }^{6}$ Dept. of Experimental Immunology, Amsterdam Infection and Immunity Institute, Amsterdam UMC, University of Amsterdam, Amsterdam, The Netherlands.

${ }^{7}$ Dept. of Nephrology, University Medical Center Groningen, University of Groningen. Groningen, the Netherlands.

${ }^{8}$ Erasmus MC transplant institute, University Medical Center Rotterdam, Department of Internal Medicine Rotterdam, the Netherlands.

${ }^{9}$ Dept. of Internal Medicine, Div. of Nephrology. Amsterdam University Medical Center - Location Amsterdam Medical Center, Amsterdam, the Netherlands.

10, 11 Center for Infectious Disease Control, National Institute for Public Health and the Environment, Bilthoven, the Netherlands.

${ }^{12-16}$ Dutch Kidney Patient Association (NVN), Bussum, the Netherlands.

\section{Authors' Contributions}

$R G$, JS and $M H$ designed the study protocol. PB, JS, RG, LM, LH, MR, PV, FB, AA $M t D, A V$, and $M H$ contributed to the protocol design. PB, JS, RG, LM, LH, MR, PV, $\mathrm{FB}, \mathrm{AA}, \mathrm{MtD}, \mathrm{AV}, \mathrm{MvdD}, \mathrm{TR}, \mathrm{MS}$ and $\mathrm{MH}$ provided intellectual content of critical importance to the study and participated in preparation of the manuscript and implementation of the study. All authors revised and approved the final manuscript. The RECOVAC collaborators contributed to the design of the consortium.

\section{Funding}

The RECOVAC consortium received funding by The Netherlands Organisation for Health Research and Development (ZonMw) and the Dutch Kidney Foundation. Peer-review has been conducted by the ZonMW committee. The consortium is endorsed by the Dutch Federation of Nephrology (NFN), Dutch Transplant Society (NTV), Dutch Kidney Patient Association (NVN) and the Dutch Kidney Foundation (Nierstichting). The RECOVAC consortium will share data with the RIVM and Netherlands Pharmacovigilance Center (LAREB) whenever deemed relevant.

\section{Availability of data and materials} Not applicable.

\section{Declarations}

\section{Ethics approval and consent to participate}

Informed consent has been given by all participants. The Medical Research Ethics Committee of the University Medical Center Groningen has granted approval to carry out this study (EudraCT nr.: 2021-001520-18). The study will be conducted according to the principles of the Declaration of Helsinki (64th WMA General Assembly, Fortaleza, Brazil, October 2013) and in accordance with the Medical Research Involving Human Subjects Act (WMO).

\section{Consent for publication}

Not applicable.

\section{Competing interests}

None declared.

\section{Author details}

${ }^{1}$ Department of Internal Medicine, Division of Nephrology, Maastricht University Medical Center, Maastricht, the Netherlands. ${ }^{2}$ CARIM school for cardiovascular disease, University of Maastricht, Maastricht, the Netherlands. ${ }^{3}$ Department of Nephrology, University Medical Center Groningen, University of Groningen, Groningen, the Netherlands. ${ }^{4}$ Department of Nephrology, Radboud University Medical Center, Nijmegen, the Netherlands. ${ }^{5}$ Internal Medicine, Nephrology and Transplantation, Erasmus MC Transplant Institute, Erasmus Medical Center, Rotterdam, the Netherlands. ${ }^{6}$ Department of Internal Medicine, University Medical Center Groningen, Groningen, the Netherlands. ${ }^{7}$ Department of Clinical Pharmacy and Pharmacology, University Medical Center Groningen, Groningen, the Netherlands. ${ }^{8}$ Department of Internal Medicine, Division of Nephrology, Amsterdam University Medical Center location Amsterdam Medical Center, Amsterdam, the Netherlands. ${ }^{9}$ Department of Nephrology and Hypertension, University Medical Center Utrecht, Utrecht, the Netherlands. ${ }^{10}$ Department of Nephrology, Maasstad Hospital, Rotterdam, the Netherlands. ${ }^{11}$ Department of Internal Medicine, Canisius Wilhelmina Hospital, Nijmegen, the Netherlands. ${ }^{12}$ Department of Medicine, Division of Nephrology, and Leiden Transplant Center, Leiden University Medical Center, Leiden, the Netherlands. ${ }^{13}$ Department of Immunopathology, Sanquin Research, Amsterdam, The Netherlands. ${ }^{14}$ Landsteiner Laboratory, Amsterdam University Medical Center, University of Amsterdam, Amsterdam, The Netherlands.

Received: 2 July 2021 Accepted: 20 January 2022

Published online: 05 February 2022

\section{References}

1. Williamson EJ, Walker AJ, Bhaskaran K, Bacon S, Bates C, Morton CE, et al. Factors associated with COVID-19-related death using OpenSAFELY Nature. 2020;584(7821):430-6.

2. Gansevoort RT, Hilbrands LB. CKD is a key risk factor for COVID-19 mortality. Nat Rev Nephrol. 2020;16(12):705-6.

3. Hilbrands LB, Duivenvoorden R, Vart P, Franssen CFM, Hemmelder MH Jager KJ, et al. COVID-19-related mortality in kidney transplant and dialysis patients: results of the ERACODA collaboration. Nephrol Dial Transplant. 2020;35(11):1973-83.

4. Jager KJ, Kramer A, Chesnaye NC, Couchoud C, Sánchez-Álvarez JE, Garneata $L$, et al. Results from the ERA-EDTA Registry indicate a high mortality due to COVID-19 in dialysis patients and kidney transplant recipients across Europe. Kidney Int. 2020;98(6):1540-8.

5. Sadoff J, Gray G, Vandebosch A, Cárdenas V, Shukarev G, Grinsztejn B, et al Safety and Efficacy of Single-Dose Ad26.COV2.S Vaccine against Covid-19. N Engl J Med. 2021

6. Baden LR, El Sahly HM, Essink B, Kotloff K, Frey S, Novak R, et al. Efficacy and Safety of the mRNA-1273 SARS-CoV-2 Vaccine. N Engl J Med. 2021;384(5):403-16

7. Polack FP, Thomas SJ, Kitchin N, Absalon J, Gurtman A, Lockhart S, et al. Safety and Efficacy of the BNT162b2 mRNA Covid-19 Vaccine. N Engl J Med. 2020;383(27):2603-15.

8. Voysey M, Clemens SAC, Madhi SA, Weckx LY, Folegatti PM, Aley PK, et al. Safety and efficacy of the ChAdOx1 nCoV-19 vaccine (AZD1222) against SARS-CoV-2: an interim analysis of four randomised controlled trials in Brazil, South Africa, and the UK. Lancet. 2021;397(10269):99-111.

9. Glenn DA, Hegde A, Kotzen E, Walter EB, Kshirsagar AV, Falk R, et al. Systematic review of safety and efficacy of COVID-19 vaccines in patients with kidney disease. Kidney Int Rep. 2021.

10. Reddy S. Vaccination in Chronic Kidney Disease. Adv Chronic Kidney Dis. 2019;26(1):72-8.

11. Ortiz A, Cozzolino M, Fliser D, Fouque D, Goumenos D, Massy Z, et al. Chronic kidney disease is a key risk factor for severe COVID-19: a call to action by the ERA-EDTA. Nephrol Dial Transplant. 2020;36. 
12. Lacson E, Argyropoulos CP, Manley HJ, Aweh G, Chin Al, Salman LH, et al. Immunogenicity of SARS-CoV-2 Vaccine in Dialysis. medRxiv. 2021; 2021.05.11:[2021.04.08.21254779 p.]. Available from: https://www.medrx iv.org/content/medrxiv/early/2021/04/13/2021.04.08.21254779.full.pdf.

13. Grupper A, Sharon N, Finn T, Cohen R, Israel M, Agbaria A, et al. Humoral Response to the Pfizer BNT162b2 Vaccine in Patients Undergoing Maintenance Hemodialysis. Clin J Am Soc Nephrol. 2021:CJN.03500321.

14. Marinaki S, Adamopoulos S, Degiannis D, Roussos S, Pavlopoulou ID, Hatzakis A, et al. Immunogenicity of SARS-CoV-2 BNT162b2 vaccine in solid organ transplant recipients. Am J Transplant. 2021; Apr 17.

15. Vogelzang EH, Loeff FC, Derksen NIL, Kruithof S, Ooijevaar-de Heer P, van Mierlo G, et al. Development of a SARS-CoV-2 Total Antibody Assay and the Dynamics of Antibody Response over Time in Hospitalized and Nonhospitalized Patients with COVID-19. J Immunol. 2020;205(12):3491-9.

16. RECOVAC. [updated 25th of May. Available from: www.recovac.nl.

17. Steenhuis M, van Mierlo G, Derksen NIL, Ooijevaar-de Heer P, Kruithof S, Loeff FL, et al. Dynamics of antibodies to SARS-CoV-2 in convalescent plasma donors. medRxiv. 2021; 2021.01.06.20249035.

18. Hodgson SH, Mansatta K, Mallett G, Harris V, Emary KRW, Pollard AJ. What defines an efficacious COVID-19 vaccine? A review of the challenges assessing the clinical efficacy of vaccines against SARS-CoV-2. Lancet Infect Dis. 2021;21(2):e26-35.

19. Schultz NH, Sørvoll IH, Michelsen AE, Munthe LA, Lund-Johansen F, Ahlen MT, et al. Thrombosis and Thrombocytopenia after ChAdOx1 nCoV-19 Vaccination. N Engl J Med. 2021.

\section{Publisher's Note}

Springer Nature remains neutral with regard to jurisdictional claims in published maps and institutional affiliations.

- fast, convenient online submission

- thorough peer review by experienced researchers in your field

- rapid publication on acceptance

- support for research data, including large and complex data types

- gold Open Access which fosters wider collaboration and increased citations

- maximum visibility for your research: over $100 \mathrm{M}$ website views per year

At BMC, research is always in progress.

Learn more biomedcentral.com/submissions 